\title{
Isolation and Characterization of a Bacteriophage Tail-Like Bacteriocin from a Strain of Rhizobium
}

\author{
WOLFGANG LOTZ AND FRANK MAYER ${ }^{1}$ \\ Institut fiir Mikrobiologie, Universität Erlangen, Erlangen, Germany
}

Received for publication 2 September 1971

\begin{abstract}
Bacterial strain 16-3 spontaneously produces a bacteriocin which inhibits the growth of closely related strain 16-2. Both strains were newly isolated from root nodules of lupines and probably belong to the species Rhizobium lupini. Production of infectious progeny of newly isolated virulent phage 16-2-4 in strain 16-2 is inhibited completely if complexes are bacteriocin-treated during the first half of the latent period. Treatment begun during the second half leads to premature lysis of complexes and inactivates only those progeny phages which were not yet fully matured at the moment of the particle-induced lysis. Examination by electron microscope of the bacteriocin enrichment revealed the presence of particles $123 \mathrm{~nm}$ in length which resemble the tails of T-even bacteriophages. Since the particles sediment together with the bactericidal activity in the sucrose gradient and adsorb specifically to bacteriocin-sensitive cells, it is concluded that they are identical with the bactericidal agent. The particles are not found attached to phage heads and cannot self-propagate; they are regarded as incomplete and are named INCO particles. INCO particles consist of a core enveloped by a contractile sheath. One end of the sheath is connected to a baseplate to which six fibers, each $32 \mathrm{~nm}$ in length, are attached. These connect the baseplate of an adsorbing particle to the cell surface. Since INCO cores are probably empty, it is concluded that specific adsorption of the particles to the bacterial surface is sufficient to inactive sensitive cells irreversibly.
\end{abstract}

Defective bacteriophages can be defined as "particles which contain either all or some of the normal phage components, but fail to form plaques on any known host, even at high multiplicities of infection" (11). In recent reviews, the morphology, production, and mode of action of such particles have been discussed $(3,11,17)$. Quite a number of defective phages from different sources can act bactericidally and thus be classified as high-molecular-weight bacteriocins (3).

The first description of bacteriocin production by a number of species within the economically important genus Rhizobium was published by Roslycky (18). Since detailed studies on the properties of rhizobiocins were not found in the literature, a bacteriocin produced by a bacterial strain newly isolated from root nodules of lupines (probably Rhizobium lupini) was examined.

In this report, the biological properties (inhibition of bacterial growth and of phage multiplication) of this bacteriocin will be described. Evidence is presented showing that the bacteri-

\footnotetext{
1 Present address: Biology Division, University of Texas at Dallas, Dallas, Tex. 75230.
}

cidal activity resides in defective phage particles resembling the tails of T-even bacteriophages of Escherichia coli. The morphology and mechanism of adsorption of these particles has been investigated.

\section{MATERIALS AND METHODS}

Bacteria. Bacterial strains 16-2 and 16-3 were isolated from root nodules of agriculturally used sweet lupines as described by Heumann (13). Both strains probably can be grouped within the genus of Rhizobium. They grow on the selective medium developed by Graham (12) for the growth of Rhizobium but do not grow on the selective medium for closely related Agrobacterium (6), nor do they show a positive reaction in the 3-ketolactose test which is typical for strains of Agrobacterium (2). In nodulation tests using lupines as host plants, strains $16-2$ and $16-3$ are presently being examined further for their relationship to the genus of Rhizobium (8). Growing cells of both strains have a generation time of about $70 \mathrm{~min}$ when aerated at $30 \mathrm{C}$ in fresh nutrient broth. From the bacteriocin-sensitive strain 16-2 an insensitive spontaneous mutant (16-2/I1) was isolated.

Phage. Phage 16-2-4 for bacterial strain 16-2 was isolated from garden compost. On a lawn of 16-2 cells, the phage produces clear plaques which are 
surrounded by a small halo and have a diameter of about $2.0 \mathrm{~mm}$. Before a high-titer phage stock was produced by the plate method (10), the phage was purified by four single-plaque passages. Its nucleic acid is presently being examined.

Enrichment of the bacteriocin. Overlay of soft agar containing cells of the bacteriocin-producing strain 16-3 was poured on nutrient plates and incubated overnight. The lawn of bacteria, which had then developed, was ultraviolet (UV)-irradiated (at 254 $\mathrm{nm}$ ) with a dose of $1,000 \mathrm{erg} / \mathrm{mm}^{2}$ to enhance the bacteriocin production and then covered with $3.0 \mathrm{ml}$ of nutrient broth. After a further overnight incubation of the plates, the bacteriocin was harvested by pouring the nutrient broth from the plates. The enrichment of the bacteriocin was obtained by three rounds of differential centrifugation. Low-speed centrifugations were carried out in a Sorvall centrifuge at $10,000 \mathrm{rev} / \mathrm{min}$ for $10 \mathrm{~min}$. High-speed centrifugations were carried out in a WKF-ultracentrifuge at $96,000 \times g$ for $6 \mathrm{hr}$ with an angle rotor. The enrichment so obtained was used for most of the experiments and was termed "standard enrichment." Bacteriocin concentrations used in the experiments are given as steps of dilution per milliliter of the standard enrichment and termed "relative concentration." The bacteriocin, suspended in buffer, was stored at $4 \mathrm{C}$ over several drops of chloroform.

Media. The medium used in these experiments contains $8 \mathrm{~g}$ of nutrient broth (Difco) per liter of water. For agar plates, $18 \mathrm{~g}$ of agar (Difco) was added to 1 liter of this medium; for agar overlays, $7 \mathrm{~g}$ of agar was added per liter of medium. Dilutions were carried out in buffer ( $p \mathrm{H}$ 7.0) containing (per liter): $7 \mathrm{~g}$ of $\mathrm{Na}_{2} \mathrm{HPO}_{4} \cdot 2 \mathrm{H}_{2} \mathrm{O}, 3 \mathrm{~g}$ of $\mathrm{KH}_{2} \mathrm{PO}_{4}$, and $4 \mathrm{~g}$ of $\mathrm{NaCl}$. Phages, as well as the bacteriocin, were suspended in buffer for storage.

General experimental conditions. All incubations were carried out at $30 \mathrm{C}$. Bacterial cultures were aerated in a roller-tube apparatus; optical density (OD) was measured at $650 \mathrm{~nm}$ in a Spectronic 20 photometer (Bausch \& Lomb). Phage multiplication was measured by single-step growth experiments by using the agar layer technique for plating (1). For the assay of plaque-formers, the bacteriocin-insensitive strain 16-2/I1 was used as indicator. This mutant has the same plating efficiency for phage $16-2-4$ as wildtype strain 16-2.

Sucrose gradient centrifugation: first run. A 0.2 $\mathrm{ml}$ amount of the enrichment of the bacteriocin was layered on a linear 5 to $20 \%(\mathrm{w} / \mathrm{v})$ sucrose gradient $(5 \mathrm{ml})$ prepared in buffer and then centrifuged for 90 $\mathrm{min}$ at $35,000 \mathrm{rev} / \mathrm{min}$ in a swing-out rotor. Fractions of five drops each were then collected from the centrifugation tube and, after addition of $2 \mathrm{ml}$ of buffer to each fraction, were examined for their OD at $280 \mathrm{~nm}$ in the Zeiss PMQ II photometer and for their bactericidal activity in a semiquantitative spot test. For this test, $0.02-\mathrm{ml}$ portions of the $10^{-1}, 10^{-2}$, and $10^{-3}$ per ml dilutions of each fraction were spotted on agar plates which had been inoculated with 16-2 bacteria by the agar layer technique (1). The plates were then incubated for $20 \mathrm{hr}$ to allow growth of the bacterial "lawn." Clear spots in this lawn indicated the presence of bactericidal activity within the respective fraction. The relative bacteriocin concentration of a fraction was judged from the step of dilution still producing a clear spot in the lawr. Fractions 3 to 7 , which contained the peak of the bactericidal activity, were pooled and centrifuged for $8 \mathrm{hr}$ at $35,000 \mathrm{rev} / \mathrm{min}$ to sediment the bacteriocin. The pellet so obtained was resuspended in buffer.

Sucrose gradient centrifugation: second run. With $0.2 \mathrm{ml}$ of the above described resuspension, the sucrose gradient centrifugation was repeated under the conditions described for the first run. To test the bactericidal activity, $0.1 \mathrm{ml}$ from each fraction was added to 0.9 $\mathrm{ml}$ of a growing 16-2 culture (cell titer $2 \times 10^{8}$ per $\mathrm{ml}$ ). After $20 \mathrm{~min}$ at $30 \mathrm{C}$, the culture was diluted at least $10^{-5}$ per $\mathrm{ml}$ in buffer and then tested for colonyformers. For each fraction the percentage of cells killed was determined in relation to the untreated control.

Electron microscopy. All preparations were carried out on carbon-coated copper grids. Negative staining (5) was performed with uranyl acetate or uranyl formate at a concentration of $2 \%$ and a $p \mathrm{H}$ of 4.8 . A drop of a suspension of bacteria or (intact or defective) phages was either mixed with an equal volume of staining solution before being transferred to the grid, or first placed on the grid, blotted with filter paper, and then negatively stained. In an alternative method, bacteria or phages were directly suspended in the staining solution on the solid medium on which they were propagated and then transferred to the grid. Electron micrographs were taken with a Jeol Jem 7 A instrument.

\section{RESULTS}

Production of a bactericidal agent. Bacterial strain 16-3 produces a bactericidal agent which inhibits growth of strain 16-2. This agent diffuses from 16-3 colonies into the surrounding medium, producing inhibition zones if the colonies are overlayered by soft agar containing 16-2 cells (Fig. 1). The inhibition zones are produced spontaneously but can be enlarged by UV irradiation of growing 16-3 colonies, indicating that the bacteriocin production is UV-inducible. Strains $16-2$ and $16-3$ must be closely related since virulent phage strains were isolated which can be propagated on both with the same efficiency of plating. In contrast to strain 16-2, cells of strain 16-3 are resistant against the bacteriocin.

Inhibition of bacterial growth. Survival of cells of strain 16-2 in the stationary phase (Fig. 2A) is affected less by increasing concentrations of the bacteriocin than that of growing (Fig. 2B) cells. The slopes of both survival curves decrease with increasing bacteriocin concentrations, indicating a heterogeneity of the cell population. The survival curve for growing cells (Fig. 2B) almost reaches a plateau for higher bacteriocin concentrations; this plateau corresponds to 


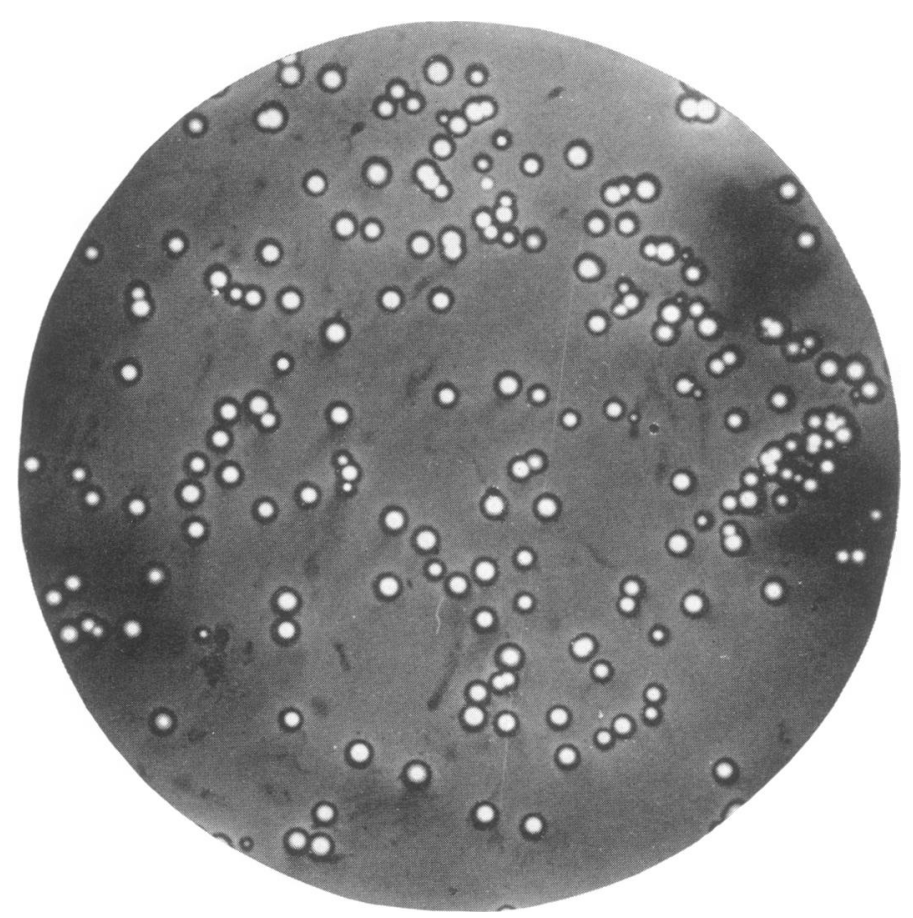

FIG. 1. Colonies of bacteriocin-producing strain 16-3 were grown on a nutrient agar plate for $70 \mathrm{hr}$ and then inactivated by chloroform vapor. After evaporation of the chloroform, plates were overlayered by soft agar containing cells of the bacteriocin-sensitive strain 16-2 and incubated for another $20 \mathrm{hr}$. Inhibition zones in the indicator lawn could then be observed around 16-3 colonies. The zones are produced by the bacteriocin, which diffuses from the colonies into the surrounding medium.

about $1 \%$ of the cells. Since in preliminary experiments cells of 10 colonies picked separately from titer plates of the "plateau" showed wildtype bacteriocin sensitivity, the resistance at higher concentrations is probably due to physiological, not genetic, differences within the cell population.

Inhibition of phage multiplication. For the experiments described below, the newly isolated virulent phage 16-2-4 for strain 16-2 was used (Fig. 3). This phage has a minimum latent period of $60 \mathrm{~min}$ and an average burst size of 80 to 90 plaque-forming units (Fig. 5A).

Bacteriocin treatment of 16-2-4-infected bacteria of strain 16-2 has marked effects on lysis. Addition of the bacteriocin early after infection prevents lysis (and, correspondingly, the production of infectious phage progeny), whereas later addition induces premature lysis (Fig. 4). The OD of the phage-infected culture (Fig. 4B) increases during the latent period at about the same rate as that of the uninfected culture (Fig. 4A). This initial increase ends about $60 \mathrm{~min}$ after infection, at which time the OD decreases sharply, reaching a lower plateau at about 180 min after infection. The sharp decrease in OD must have been caused by lysis of the complexes at the end of the latent period. This curve (Fig. 4B) will be referred to as "control."

The initial increase of the OD mentioned above is inhibited completely when the bacteriocin is added simultaneously with the phages to a growing culture of bacteria (Fig. 4C); also, there is almost no decrease in OD following the first $60 \mathrm{~min}$ after infection, indicating that lysis of most complexes is inhibited completely. Addition of the agent $30 \mathrm{~min}$ after phage infection results in a premature decrease of the OD followed by a plateau (Fig. 4D) which lies above that of the control (B). In this case, treatment with the bactericidal agent must have induced premature lysis in part of the complexes while inhibiting lysis of the rest of the infected cells. In contrast, addition of the agent 60 min after infection, at the end of the latent period, leads to an immediate decrease of the OD (Fig. 4E) which is steeper than that of the control; the lower plateau of this curve lies only slightly above that of the control. Here, the agent must have induced premature lysis in most of the complexes. Correspondingly, addition of the agent $90 \mathrm{~min}$ after infection, at which time the decrease of the OD has already 


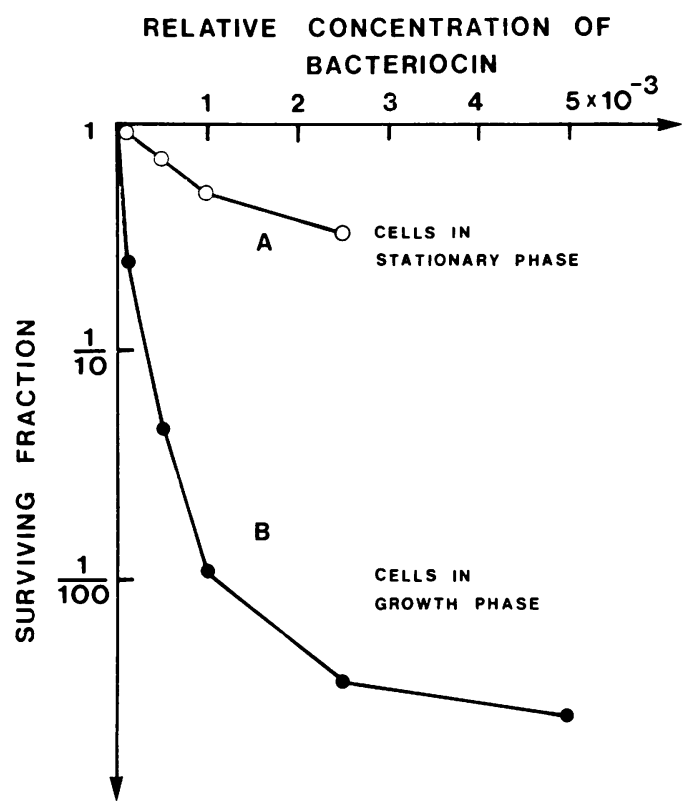

FIG. 2. Survival of 16-2 cells in stationary $(A)$ and early growth phase $(B)$ after bacteriocin treatment. $C$ Cultures $A$ and $B$ (at a cell titer of $2 \times 10^{8}$ per $\mathrm{ml}$ ) were treated for $15 \mathrm{~min}$, respectively, with increasing concentrations of the bacteriocin and then diluted at least $10^{-4}$ in buffer before they were plated for colony formers.

begun in the control (B), results in an accelerated decrease (Fig. 4F).

It can be concluded (Fig. 4) that the fraction of complexes lysing prematurely through the action of the bacteriocin increases with the time after infection at which the latter was added. In a further experiment (not shown in Fig. 4), the bacteriocin was added to complexes at different times during the first half of the latent period, and it was found that premature lysis can be induced in a small fraction of the infected bacteria as early as 10 to $15 \mathrm{~min}$ after infection. The effect of the bacteriocin on lysis seems to be similar to that of cyanide on T4-infected $E$. coli bacteria which are lysis-inhibited or prematurely lysed, depending on whether cyanide is added early or late during the latent period (9).

To test whether infectious progeny phages were set free by the bacteriocin-induced lysis of complexes, samples from a phage-infected bacterial culture were treated for only $10 \mathrm{~min}$, respectively, with the bactericidal agent before being diluted and plated for plaque formers. Figure 5B shows that the growth curve for phages from such cultures rises about $30 \mathrm{~min}$ ahead of that of the untreated control (Fig. 5A), indicating that infectious phage particles are set free prematurely and that intracellular phage maturation begins about $30 \mathrm{~min}$ after infection.
The latter can also be deduced from Fig. 5C, which shows the growth curve for phages from chloroform-treated complexes. It is probable that bacteriocin addition during the second half of the latent period inactivates only those intracellular progeny phages which were not fully mature at the moment of the premature lysis.

Presence of a defective bacteriophage in the enrichment of the bacteriocin. Samples of the enrichment of the bacteriocin were examined by electron microscopy. They were found to contain particles resembling the tails of T-even bacteriophages of $E$. coli (Fig. 6). The particles consist of an inner core which is enveloped by a sheath. The latter exists either in the extended (EXT) or contracted (CON) state. Since the enrichment does not contain any plaque formers for 80 strains of Rhizobium and Agrobacterium examined, the particles probably cannot self-propagate. They are not found attached to phage heads. They are regarded as incomplete for these reasons and are named INCO particles.

Membranous structures which resemble empty phage heads can be observed in the enrichment of these particles (Fig. 6M). These structures are found less frequently in the enrichment than the tail-like INCO particles (only about $1 \%$ of the latter as judged from electron microscopic observations). Since it was possible that the tail-like structures were originally attached to phage heads which detached from them during the
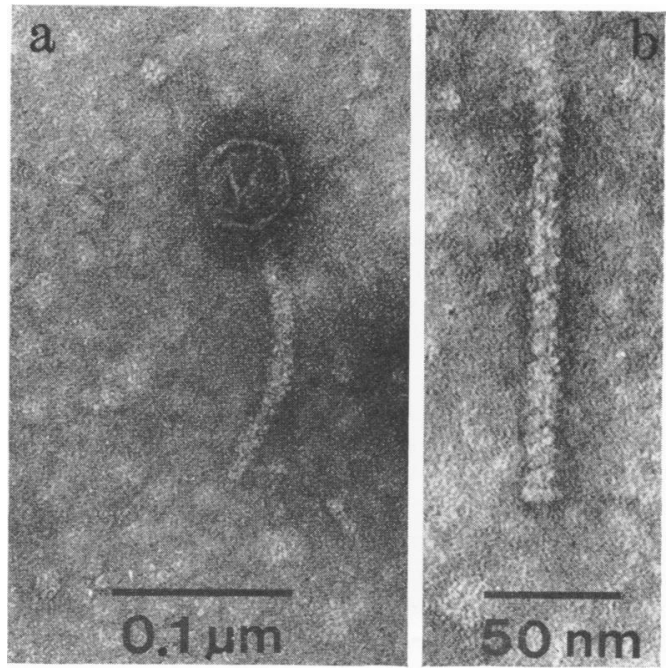

FIG. 3. Electron micrograph of bacteriophage 16-2-4 of bacterial strain 16-2 (a). This phage has a flexible tail, $140 \mathrm{~nm}$ in length, which shows a helical structure (b). The phage head is hexagonal in outline and has a diameter of $50 \mathrm{~nm}$. Negative staining was with uranyl acetate. 


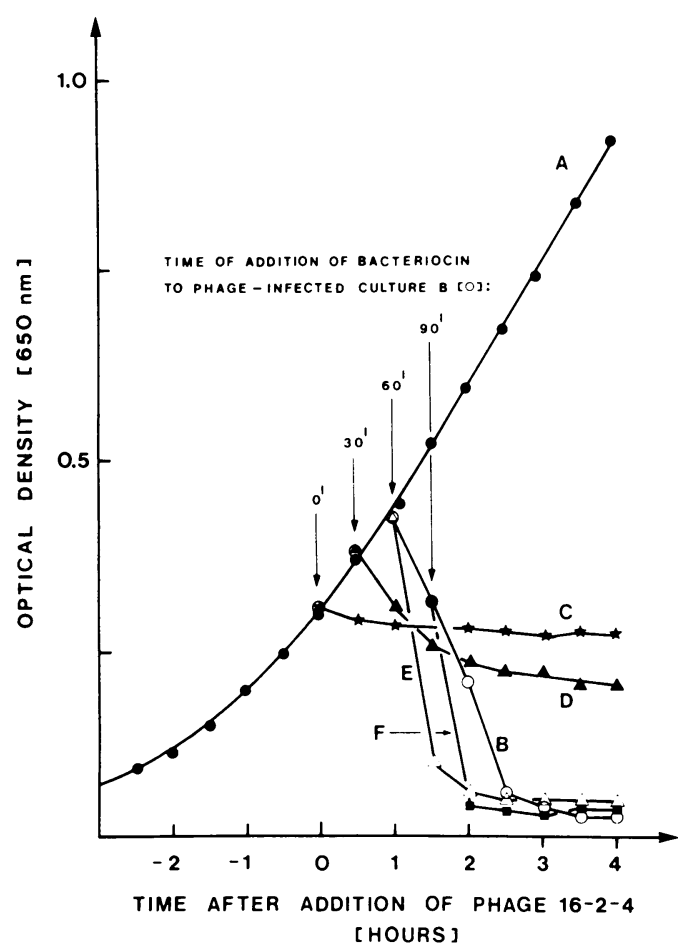

FIG. 4. Effect of bacteriocin treatment on the lysis of 16-2-4 phage-infected cells of bacterial strain 16-2. Cells of this strain were passed from a stationary overnight culture into fresh broth and incubated. After $3 \mathrm{hr}$, this new culture (titer $2 \times 10^{8}$ per $\mathrm{ml}$ ) had reached the early growth phase and was divided into six parallel cultures $(A$ to $F)$. Neither phage nor bacteriocin was added to culture $A(O)$, whereas phage 16-2-4 was added at time zero to cultures $B$ to $F$ (input multiplicity 10). No bacteriocin was added to culture $B(O)$ : to cultures $C$ to $D$, it was added (at a final relative concentration of $10^{-2}$ per $\mathrm{ml}$ ) at increasingly later times (see arrows); to culture $C(\star)$, it was added simultaneously with the phages; to cultures $D, E, F(\Delta, \triangle$, 口), it was added 30, 60, and 90 min, respectively, after phage addition. The extinction of the cultures was measured in relation to the time of incubation after phage addition.

negative-staining procedures, the particles were prefixed with glutaraldehyde before negative staining.

For this experiment, about $2 \times 10^{9}$ cells were sedimented from a growing culture of strain 16-2 and resuspended in $1 \mathrm{ml}$ of the undiluted enrichment of the bacteriocin. After 20 min (at $30 \mathrm{C}$ ) for INCO-particle adsorption, glutaraldehyde was added to the suspension to give a final concentration of $1 \%$. After $3 \mathrm{hr}$ at $4 \mathrm{C}$ for fixation, the bacteria were washed four times in buffer and finally resuspended in $1 \mathrm{ml}$ of buffer. Samples from this suspension were negatively stained with uranyl acetate and checked for adsorbed particles in the electron microscope. It was found that the surface of all bacterial cells from these samples was completely covered by adsorbed INCO particles. Since the latter were not found connected to phage heads, it is probable that they already are released from 16-3 cells as "headless" particles.

The bacteriocin from the enrichment was purified by two successive runs on a linear sucrose gradient (Fig. 7). This figure shows only one major peak for the bactericidal activity. This peak coincides with the peak for the OD which is relatively small, indicating that material was lost (or destroyed) through the repeated run over the gradient.

Samples from the 17 fractions obtained by the second run were examined with the electron microscope. In fractions 6 and 7, which contained the peak of the bactericidal activity, only INCOspecific structures were found (Fig. 8); they contained more INCO particles and substructures of

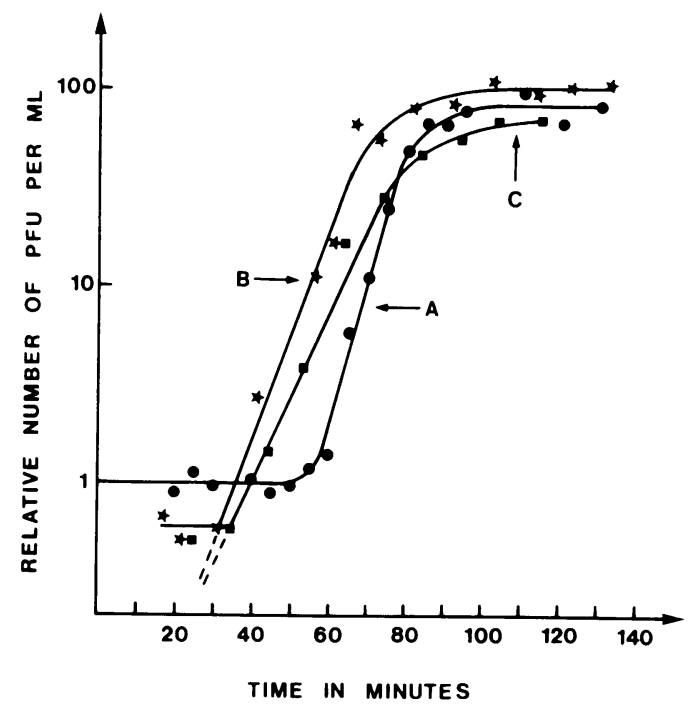

FIG. 5. Liberation of phage progeny from complexes prematurely lysed either by treatment with the bacteriocin (B) or with chloroform $(C)$. A growing culture of bacterial strain 16-2 (cell titer $2 \times 10^{8}$ per $\mathrm{ml}$ ) was infected with phage 16-2-4 at an input multiplicity of one. After 15 min for phage adsorption, the mixture was diluted $10^{-5}$ into fresh broth and incubated further. From this culture, samples were taken and plated for plaque formers at times indicated (O) to give the singlestep growth curve of the control $(A)$. Samples taken at times indicated $(\star$ ) were treated with bacteriocin (final relative concentration $10^{-2}$ per $\mathrm{ml}$ ) for $10 \mathrm{~min}$, respectively, before being plated for plaque formers $(B)$, whereas samples taken at times indicated (ם) were treated with chloroform ( 6 drops per ml of sample) for 15 min, respectively, before being diluted and plated (C). The number of plaque formers from $A, B$, and $C$ was related to the time after phage addition. 


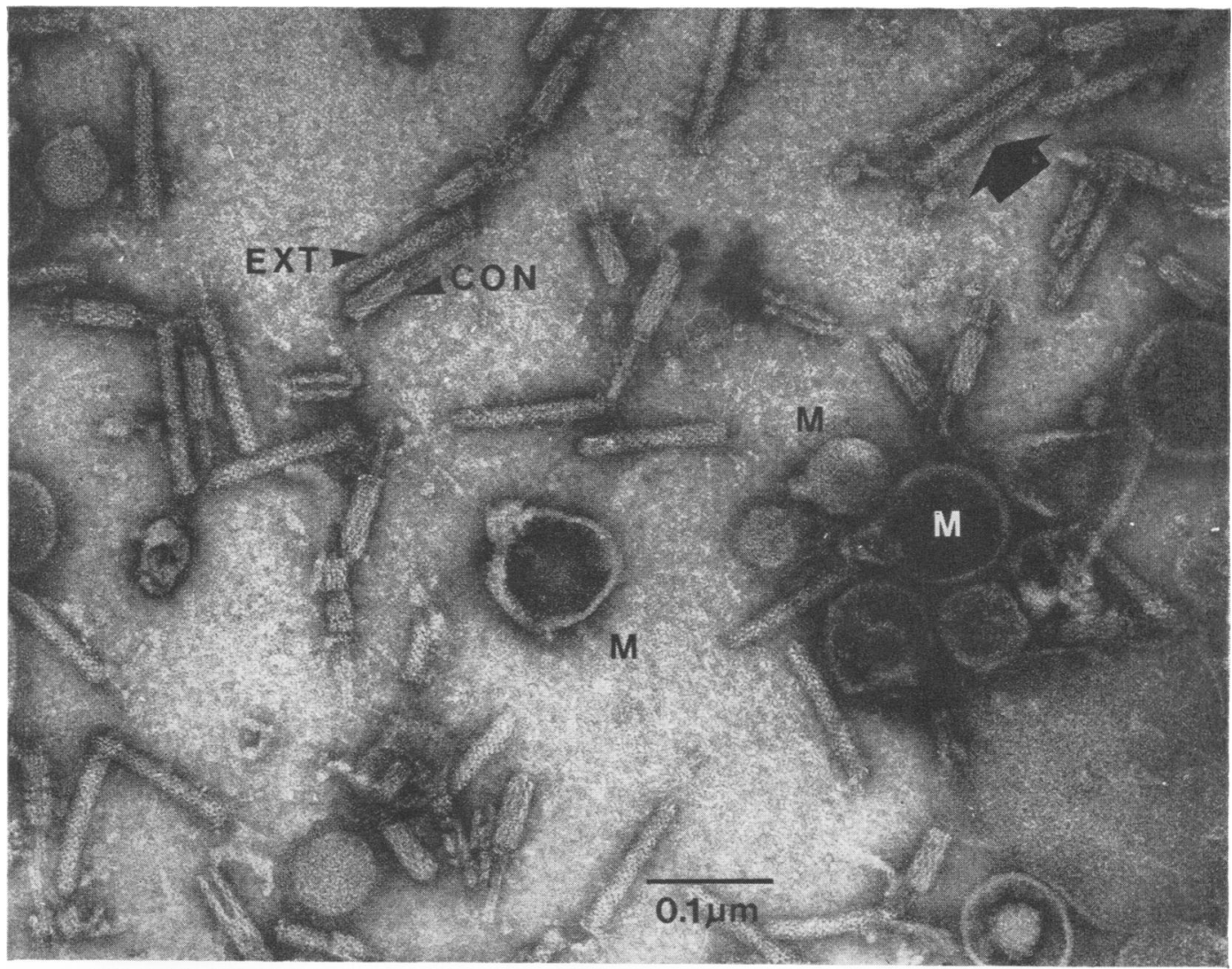

FIG. 6. Electron micrograph of a sample taken from the enrichment fraction of the bacteriocin produced by strain 16-3. In addition to the tail-like INCO particles, which can be observed in the extended (EXT) and contracted $(C O N)$ state, structures can be seen resembling empty phage heads $(M)$. Negative-staining solution has entered more than half of the core of a particle with an extended sheath (see arrow). Negative staining was with uranyl acetate.

the latter than any of the other fractions. Most of the particles found in any of the 17 fractions had contracted sheaths.

The sheath contraction could be a consequence of the purification of INCO particles if this purification resulted in the loss of some structural component of the particles necessary for the maintenance of the extended state (14). The negative-staining procedure is probably not the primary cause for this contraction since most of the negatively stained particles from unpurified enrichments were found to have extended sheaths.

In Fig. 8, many contracted sheaths without cores and isolated cores can be seen. The dissociation of particles from either purified or unpurified (Fig. 13) preparations into these substructures seems to be facilitated by sheath contraction.

Structure of INCO particles. Most INCO

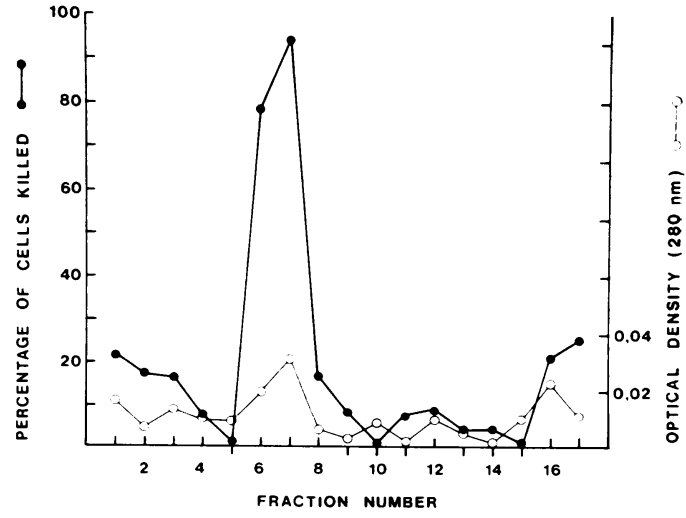

FIG. 7. Purification of the bacteriocin by sucrose gradient centrifugation. Fractions from a first run of the bacteriocin on a linear gradient, which contained the peak of the bactericidal activity, were pooled and rerun. Symbols: (O) percentage of cells killed by the bacteriocin, (O) optical density at $280 \mathrm{~nm}$. 


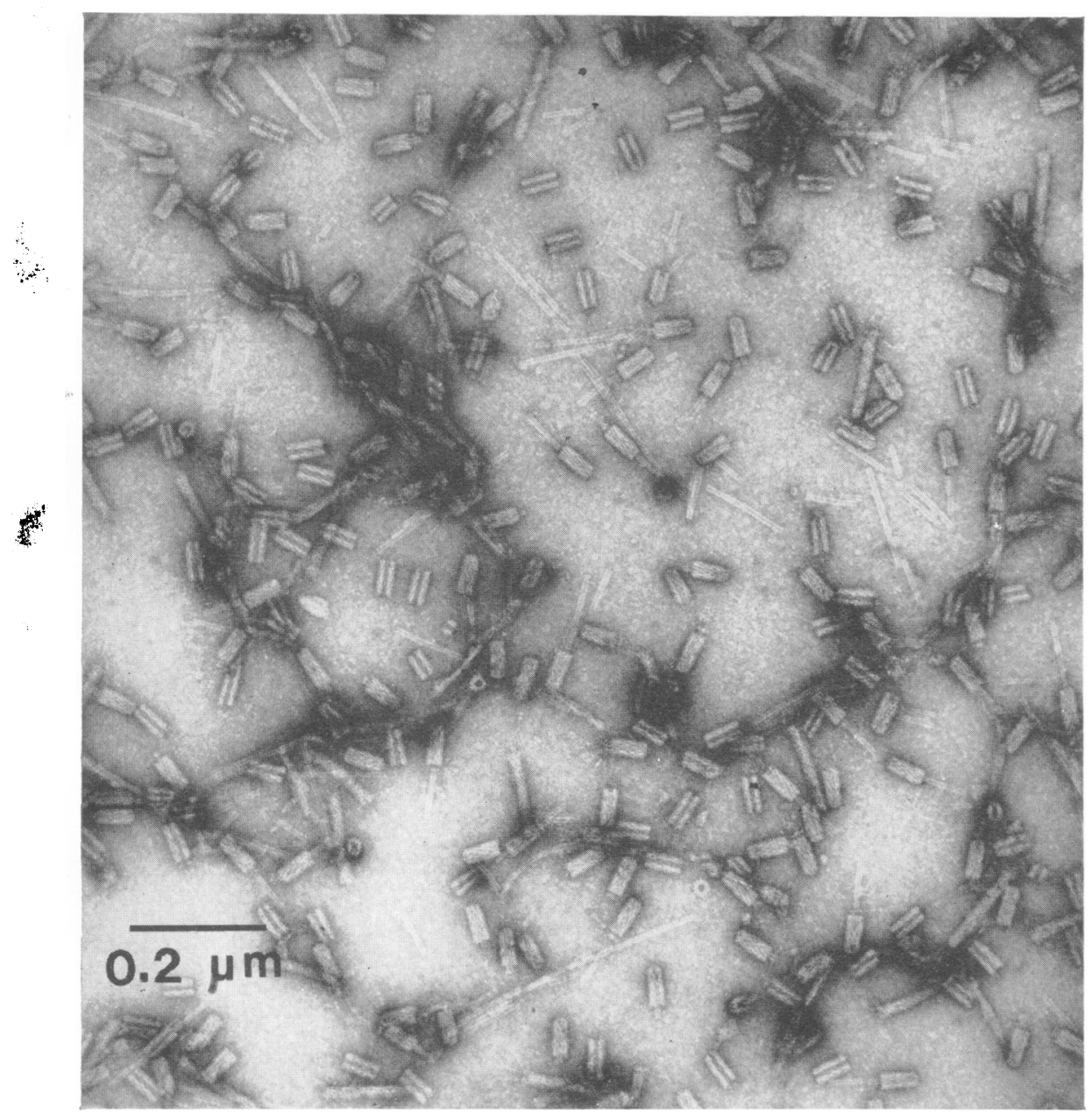

Fig. 8. Electron micrograph of a typical sample taken from fraction 7 of the sucrose gradient centrifugation shown in Fig. 7. Negative staining was with uranyl acetate.

particles with extended sheaths have an overall length of $123 \mathrm{~nm}$ (Fig. 9b). Occasionally, particles having a length of $136 \mathrm{~nm}$ were found in the enrichment fraction of the bacteriocin (Fig. 9c), whereas only once was one structure observed having a length of $104 \mathrm{~nm}$ (Fig. 9a). A baseplate (B) with spike-like structures is attached to one end of the sheath; at the opposite end, a short structure defined as "end piece" (E) protrudes from the sheath. A region (R) of seemingly structural heterogeneity is seen on almost all particles with an extended sheath; this is an important observation and will be discussed below. Further dimensions of INCO particles with extended sheaths are given in Fig. 18.

Often a short, dark line about $32 \mathrm{~nm}$ long extends from the end piece of a particle to its core although the rest of the core looks solid (Fig. 9b,c; see also Fig. 6). This observation is made on particles with an extended sheath. The line must have been produced by entering electron-dense negative-staining solution. In Fig. 6, this solution has entered more than half of the core of a particle with an extended sheath (see arrow), whereas in Fig. 10 the core of such a particle is penetrated by it completely.

In Fig. 11, two INCO particles with contracted sheaths are shown. Such particles have an overall length of $119 \mathrm{~nm}$. Contraction of the sheath (S) results in the protrusion of the core (C). A baseplate (B) is connected to the contracted sheath. To the plate of one of the particles, a fiber (F) is attached which seems to carry two "spherical" bodies (SP) at its free end. From the particle shown in Fig. 12, it can be concluded that six fibers are attached to a baseplate: four of these are visible on the micrograph and the remaining two are probably obscured by the 


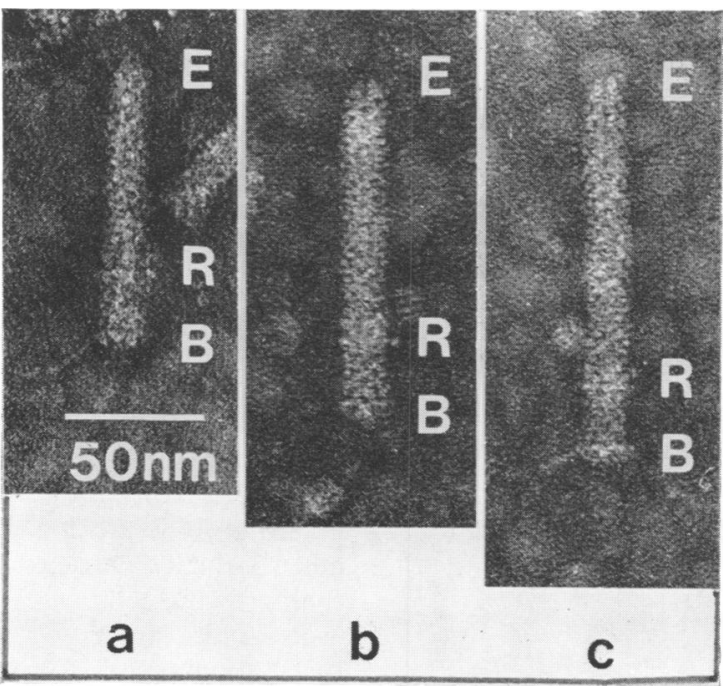

FIG. 9. Electron micrograph showing extended INCO particles of differing length: (a) $104 \mathrm{~nm},(\mathrm{~b}) 123 \mathrm{~nm},(\mathrm{c})$ $136 \mathrm{~nm}$. All particles have an outer diameter of $16.5 \mathrm{~nm} . E=$ end piece, $B=$ baseplate with spike-like structures, $R=$ region of seemingly structural heterogeneity of the sheath. Negative staining was with uranyl formate.

core. The fibers of this particle also seem to carry the mentioned bodies at their free ends. Possibly, two bodies are attached to the free end of the fiber marked by the arrow. Such bodies are difficult to distinguish and have an average diameter of only $3.7 \mathrm{~nm}$.

The particles of Fig. 11 and 12 display a region of "structural discontinuity" between the baseplate and the contracted sheath not observable on particles with extended sheaths (Fig. 9; see also Fig. 6). The core is probably not the

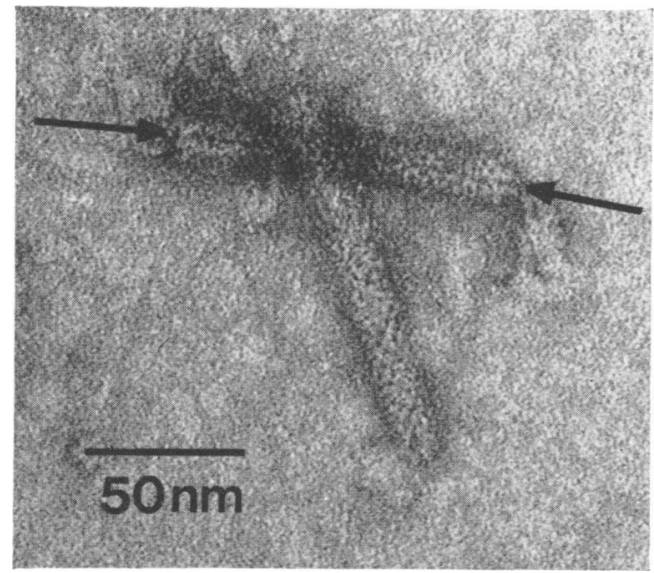

FIG. 10. Electron micrograph of INCO particles with extended sheaths. Negative-staining solution has entered the core of one of the particles completely, producing a dark line along its length axis (see arrows). Negative staining was with uranyl formate. only link between these components since additional structures can be observed within this region. In contrast to cores from particles with extended sheaths, those from particles with contracted sheaths (Fig. 11, 12) are completely penetrated by negative-staining solution which produces a dark line along their length axis having a diameter of 2.0 to $2.5 \mathrm{~nm}$.

The sheath diameter of INCO particles increases upon sheath contraction (compare particles from Fig. 13 for example) from an average value of $16.5 \mathrm{~nm}$ (uncontracted) to $24.0 \mathrm{~nm}$. The diameter of the baseplate also increases upon sheath contraction from $18.5 \mathrm{~nm}$ to $24.5 \mathrm{~nm}$. Further dimensions of INCO particles with contracted sheaths are given in Fig. 18.

Contracted as well as extended sheaths show a helical structure (Fig. 13; see arrows). In Fig. 14, a contracted sheath having a 12 -fold rotation symmetry (see marked angle of $30^{\circ}$ ) is seen in axial view. Further examination of particle fine structure should show whether the rotation symmetry of extended sheaths and of baseplates is sixfold. The structure $\left(\mathrm{B}^{\prime}\right)$ shown in Fig. 11 could be an isolated baseplate with a sixfold rotation symmetry.

Orientation of fibers and adsorption of INCO particles. In contrast to INCO particles with contracted sheaths, those with extended sheaths have at least some of the fibers folded up against the latter (Fig. $\left.15 \mathrm{~F}^{\prime}\right)$. It is possible that the region of seemingly structural heterogeneity of the sheath mentioned above (Fig. 9R) is produced by the spherical bodies of fibers folded up against 


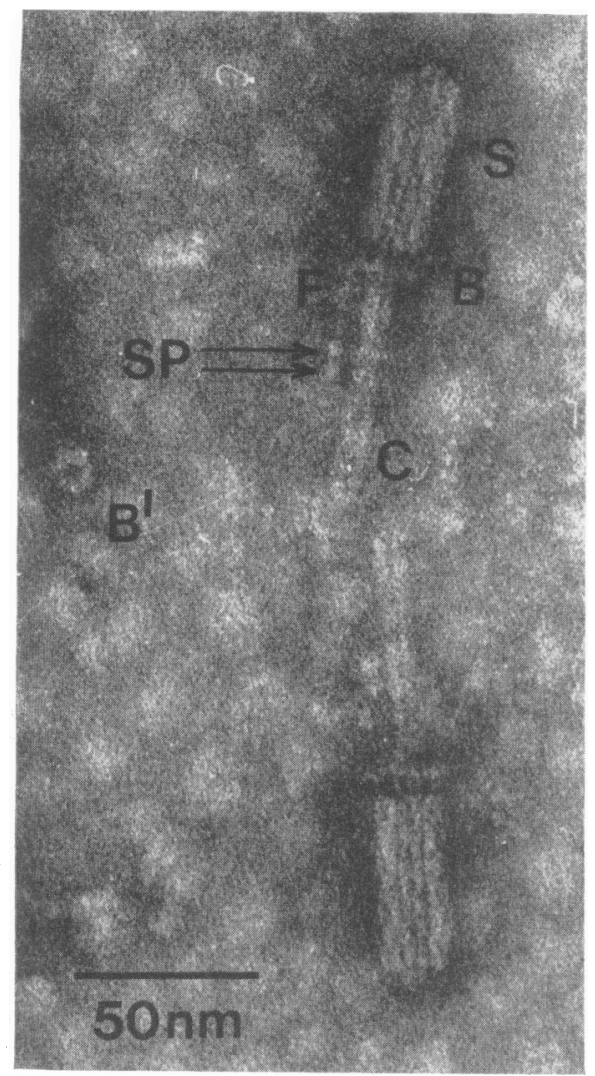

FiG. 11. Electron micrograph of INCO particles with contracted sheaths. Contraction of the sheath $(S)$ resulted in the protrusion of the core $(C)$. A fiber $(F)$ carrying possibly two spherical bodies $(S P)$ at its free end can be observed attached to the baseplate $(B)$ of one of the particles. The structure $B^{\prime}$ could be an isolated baseplate. Negative staining was with uranyl formate.

the extended sheath. The length of the fibers and the distance between this region and the baseplate are identical $(32 \mathrm{~nm})$. Correspondingly, this region cannot be observed on particles with a contracted sheath, since fibers of the latter are splayed out from the baseplate (Fig. 12).

INCO particles can adsorb to the surface of growing cells of strain 16-2 (Fig. 16). Adsorption results in the contraction of the sheath, exposing the core which seems to be in contact with the cell wall (Fig. 17, see arrow). Occasionally structures which resemble empty phage heads were found attached to the cell surface (Fig. 16, see arrow).

If the adsorption experiment described (see legend of Fig. 16 for methods) was carried through with stationary instead of growing cells, generally fewer INCO particles were found attached to the bacterial surface. No particles were found adsorbed if growing cells of strain 16-3 were used in such an experiment.

\section{DISCUSSION}

Identification of the bactericidal agent. For the reasons listed below, it is probable that the bactericidal activity resides in the INCO particles.

(i) The particles and the bactericidal agent sediment together in the ultracentrifuge during enrichment procedures. (ii) They band together with the bactericidal activity in the sucrose gradient. (iii) INCO particles adsorb to cells of the bacteriocin-sensitive strain 16-2 but not to those of the resistant strain 16-3. (iv) Stationary cells of strain 16-2 are less bacteriocin-sensitive and adsorb INCO particles probably less efficiently than growing cells.

Rhizobial strain 16-3, which produces the particles spontaneously, must be lysogenic for an INCO prophage. Whether this prophage also codes for the isolated phage head-like structures found in the enrichment is still unclear.

Structure and mechanism of adsorption of INCO particles. INCO particles resemble phage tail-like pyocins $(3,4,14,15)$ and are also structurally similar to the defective phage particles of Proteus mirabilis (21) and Proteus vulgaris (7). Description of defective phage production in $R$. lupini was not found in the literature. Models of INCO particles with an extended and a contracted

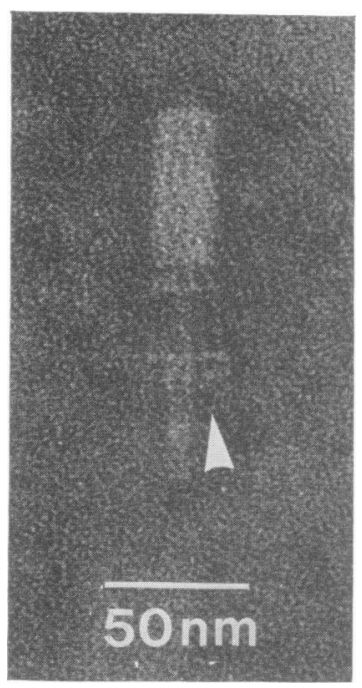

FIG. 12. Electron micrograph of an INCO particle with a contracted sheath. Probably six fibers are attached to the baseplate of the particle, four of them being observable on the micrograph and the remaining two being obscured by the core. Two spherical bodies are probably attached to the free end of the fiber marked by the arrow. Negative staining was with uranyl acetate. 


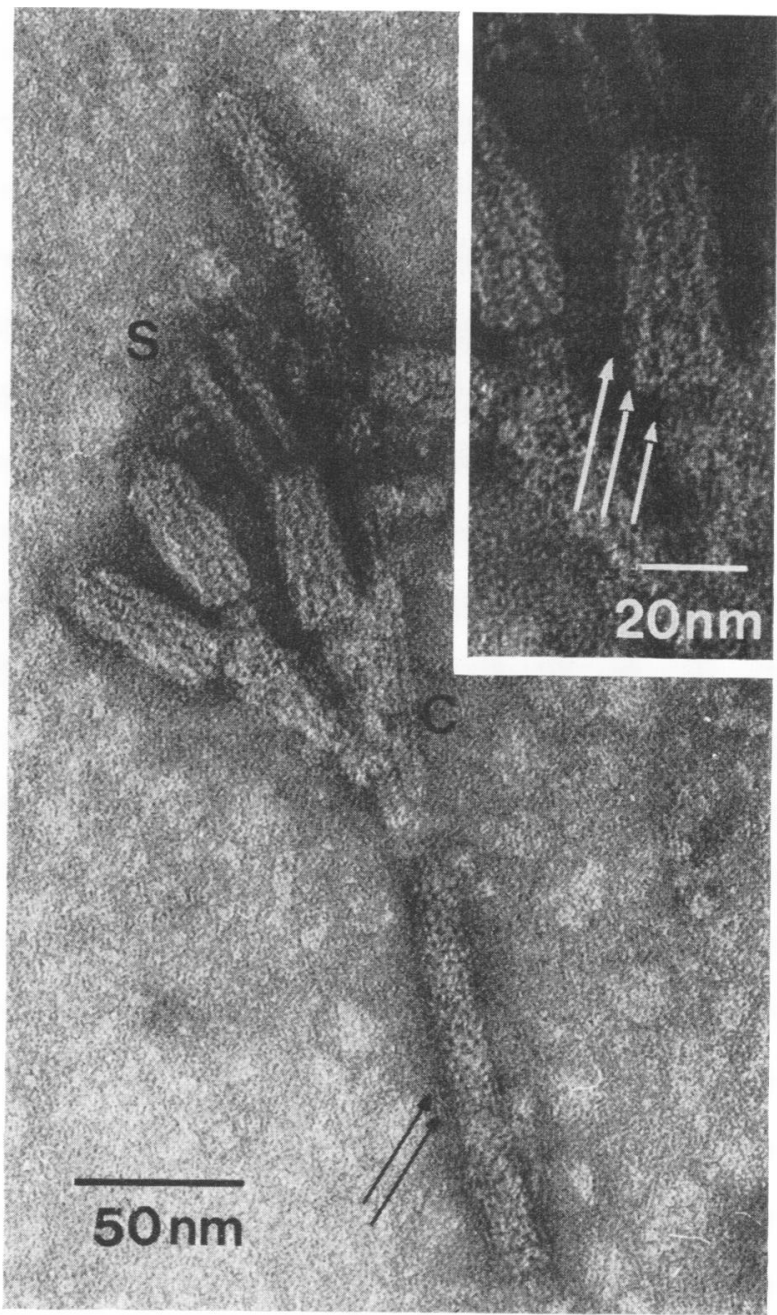

FIG. 13. Electron micrograph of INCO particles with extended or contracted sheaths. In both cases, the helical structure of the sheath can be observed (see arrows). $S=$ sheath without core, $C=$ free core (with an inner diameter of 2.0 to $2.5 \mathrm{~nm}$ ). Negative staining was with uranyl formate.

sheath, respectively, are shown in Fig. 18. The dimensions of the substructures, given in nanometers, were obtained from electron micrographs of negatively stained particles.

It is assumed that originally all six fibers of a particle with an extended sheath are folded up against the sheath (Fig. 18a) and that forces employed on the particles by procedures of enrichment or negative staining, or both, result in the "downward" orientation of some of the fibers. It is further postulated that each fiber carries at least one and possibly two "spherical" bodies at its free end. Higerd et al. (14) occasionally observed "terminal fiber knobs" having a diameter of $1 \mathrm{~nm}$ on both contracted and extended forms of pyocin particles.
The following adsorption mechanism is proposed for INCO particles. (i) The fibers of INCO particles are folded up against the extended sheath and adsorption is triggered by a specific contact between the baseplate of a particle and the cell surface. This contact leads to a structural change of the plate. Such a change is indicated by the larger diameter (about $6 \mathrm{~nm}$ ) of baseplates from particles with contracted sheaths compared to corresponding diameters from particles with extended sheaths. (ii) The structural change of the baseplate results in the "downward" orientation of the fibers which now attach by their tips to specific receptors of the cell wall. (iii) In analogy to the results of Simon and Anderson (20), it is assumed that the next 
step in the adsorption process, the contraction of the sheath, is also triggered by the structural change of the baseplate. Since the baseplate is now bound to the cell surface by the fibers, this contraction results in the penetration of the cell wall by the core.

According to this mechanism, which is regarded as a working hypothesis, the distance between the cell surface and the baseplate of an adsorbed INCO particle should correspond to the length $(32 \mathrm{~nm})$ of its fibers. Measurements taken from the electron micrograph of Fig. 17 indicate an average distance of only $29 \mathrm{~nm}$. Artifacts in

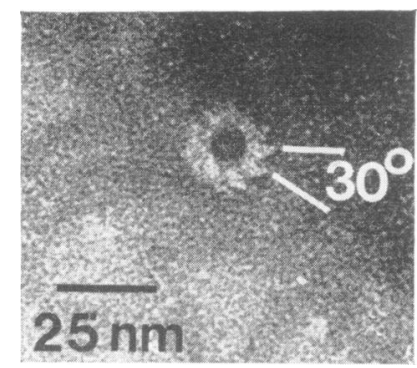

FIG. 14. Electron micrograph of a contracted INCO sheath in axial view having a 12-fold rotation symmetry (see marked angle of $30^{\circ}$ ). The outer diameter of the contracted sheath (see model b of Fig. 18) was determined from this photo. Negative staining was with uranyl formate. negative staining as described by Simon and Anderson (19) might result in measuring a distance smaller than $32 \mathrm{~nm}$.

Mode of action of the bactericidal agent. If INCO particles harbor the bactericidal agent,

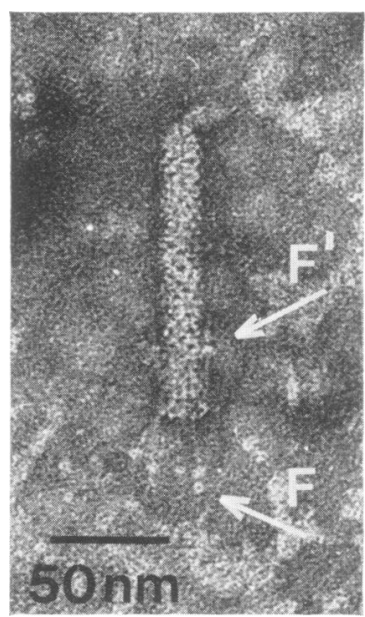

FIG. 15. Electron micrograph of an INCO particle having an extended sheath. Most of the fibers $(F)$ attached to its baseplate are extended outward from the plate, whereas one fiber $\left(F^{\prime}\right)$ seems to be folded up against the extended sheath. Negative staining was with uranyl formate.

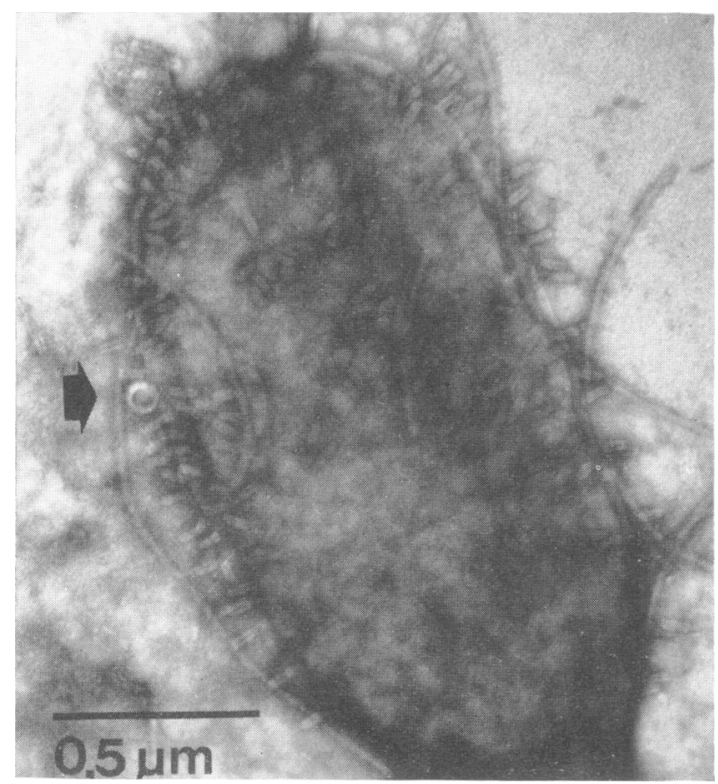

FIG. 16. Electron micrograph of INCO particles adsorbed to a cell from a growing culture of strain 16-2. This culture (cell titer $2 \times 10^{8} \mathrm{per} \mathrm{ml}$ ) was treated with the bacteriocin ( final "relative concentration" $2 \times 10^{-2}$ per ml) for 20 min to allow particle adsorption and then washed four times in buffer. A membranous structure resembling an empty phage head seems to be attached to the cell (see arrow). Negative staining was with uranyl acetate. 


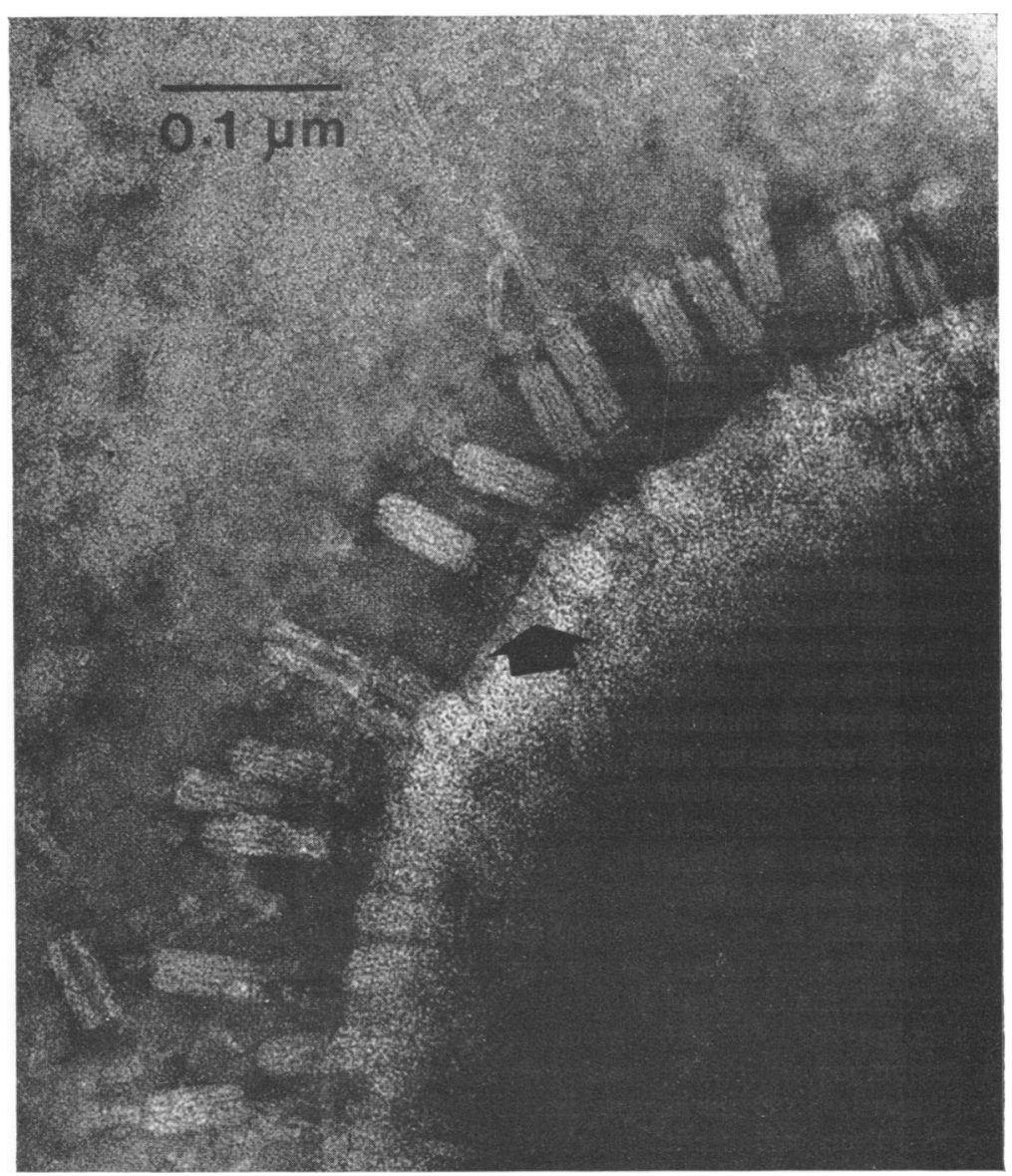

FIG. 17. Electron micrograph of INCO particles adsorbed to the surface of a cell from a growing culture of strain 16-2. Details as in Fig. 16.

their specific adsorption to sensitive cells very probably is a prerequisite for their killing action. Stationary cells of strain 16-2 might be less bacteriocin-sensitive because they adsorb the particles less efficiently than growing cells. The survival curves of Fig. 2 pass through zero point and seem to be linear for low bacteriocin concentrations, indicating that the killing of cells with the particles, as that with pyocin-R (16), is a singlehit process.

Since it was shown by preliminary experiments that bacteriocin treatment of uninfected cells does not lead to lysis, it is probable that an additional phage-specific function is necessary to induce it in the complexes. It is possible that this function corresponds to the synthesis of a phagespecific lysozyme and that the bacteriocin itself does not possess lytic activity.

The question arises as to whether the cores of INCO particles with extended sheaths are filled with a bactericidal substance which can be injected into sensitive cells upon particle adsorption. We assume this net to be the case and propose that the cores of such particles are empty (although they appear solid on electron micrographs). The cores of particles with extended sheaths are probably open to entering negative-staining solution only at the end piece, while being closed at the opposite end which is in contact with the baseplate. Since the staining solution can enter these particles only from one side, it dces not penetrate very far into them. In contrast, contraction of the sheath might result in the opening of the core at the opposite end, enabling the solution to enter from both sides, which results in cores completely filled with the stain.

Bradley and Dewar (4) observed both solid and hollow cores in pyocin particles with contracted sheaths and suggested that the material 


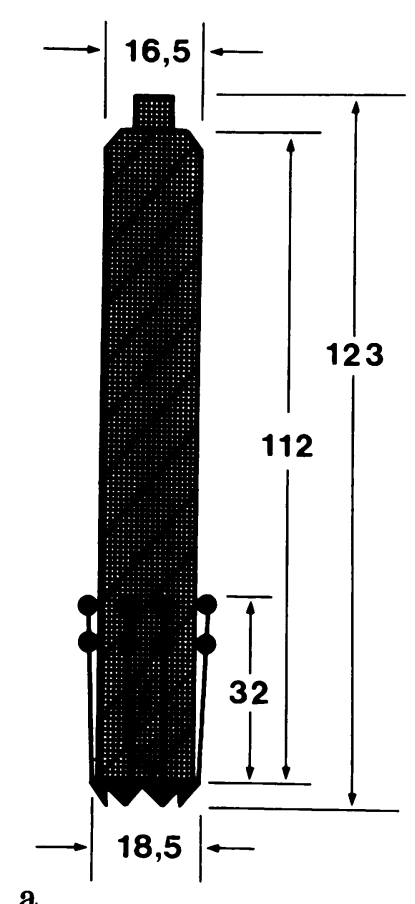

a

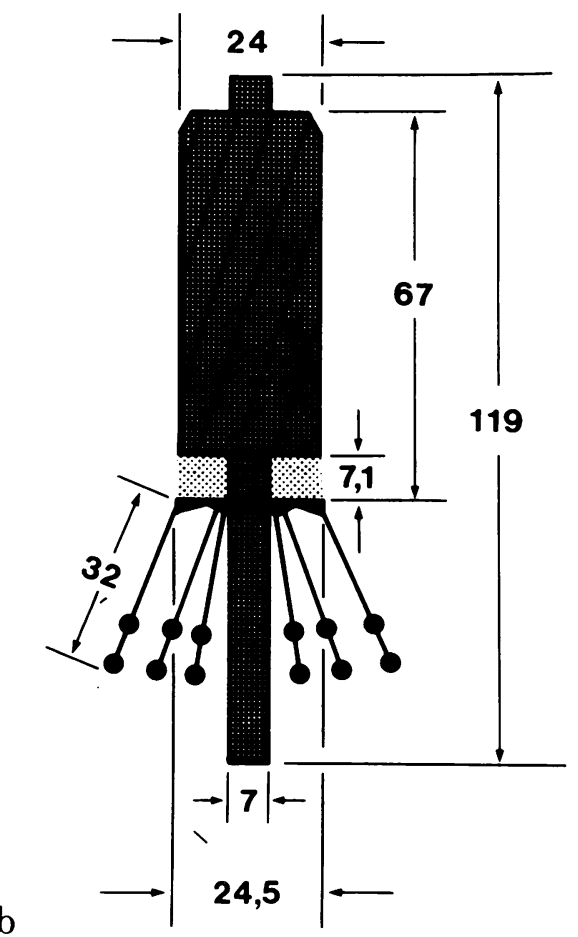

FIG. 18. Model of INCO particles with an extended (a) and a contracted (b) sheath, respectively. Dimensions are in nanometers.

in the solid cores might be a short strand of nucleic acid (probably deoxyribonucleic acid), whereas we observed only empty cores in INCO particles with contracted sheaths. If INCO particles contained nucleic acid material responsible for their bactericidal action, this material must be extremely UV-resistant, since preliminary experiments showed that the free particles (suspended in buffer) could be UV-irradiated (at $254 \mathrm{~nm}$ ) with a dose of $6,000 \mathrm{erg} / \mathrm{mm}^{2}$ without reducing their bactericidal activity.

According to the proposed adsorption mechanism, the following INCO substructures could harbor the bactericidal activity and inactivate cells upon their contact with the bacterial surface: the baseplate, the one end of the core, and the free ends of the fibers.

\section{ACKNOWLEDGMENTS}

We thank W. Heumann for his helpful comments, B. Moritz B. Görg, A. Poy, I. Bamberger, F. Schöffl for excellent technical assistance, and B. Bruton for reviewing the manuscript.

This work was supported by the Deutsche Forschungsgemeinschaft and the Stiftung Volkswagenwerk.

\section{LITERATURE CITED}

1. Adams, M. H. 1959. Bacteriophages. Interscience Publishers, Inc., New York.
2. Bernaerts, M., and J. De Ley. 1963. A biochemical test for crown-gall bacteria. Nature (London) 197:406-407.

3. Bradley, D. E. 1967. Ultrastructure of bacteriophages and bacteriocins. Bacteriol. Rev. 31:230-314.

4. Bradley, D. E., and C. A. Dewar. 1966. The structure of phagelike objects associated with non-induced bacteriocinogenic bacteria. J. Gen. Microbiol. 45:399-408.

5. Brenner, S., and R. W. Horne. 1959. A negative staining method for the high resolution electron microscopy of viruses. Biochim. Biophys. Acta 34:103-110.

6. Clark, A. G. 1969. A selective medium for the isolation of Agrobacterium species. J. Appl. Bacteriol. 32:348-351.

7. Coetzee, H. L., H. C. deKlerk, J. N. Coetzee, and J. A. Smit. 1968. Bacteriophage-tail-like particles associated with intraspecies killing of Proteus vulgaris. J. Gen. Virol. 2:29-36.

8. Dixon, R. O. D. 1969. Rhizobia. (With particular reference to relationships with host plants). Annu. Rev. Microbiol. 23: 137-158.

9. Doermann, A. H. 1952. The intracellular growth of bacteriophages. I. Liberation of intracellular bacteriophage T4 by premature lysis with another phage or with cyanide. J. Gen. Physiol. 35:645-656.

10. Eisenstark, A. 1967. Bacteriophage techniques, p. 449-524. In $\mathbf{K}$. Maramorosch and $\mathbf{H}$. Koprowski (ed.), Methods in virology, vol. I. Academic Press Inc., New York.

11. Garro, A. J., and J. Marmur. 1970. Defective bacteriophages. J. Cell. Physiol. 76:253-264.

12. Graham, P. H. 1969. Selective medium for growth of Rhizobium. Appl. Microbiol. 17:769-770.

13. Heumann, W. 1968. Conjugation in starforming Rhizobium lupini. Mol. Gen. Genet. 102:132-144.

14. Higerd, T. B., C. A. Baechler, and R. S. Berk. 1969. Morpho- 
logical studies on relaxed and contracted forms of purified pyocin particles. J. Bacteriol. 98:1378-1389.

15. Ishii, S., Y. Nishi, and F. Egami. 1965. The fine structure of a pyocin. J. Mol. Biol. 13:428-431.

16. Kageyama, M., K. Ikeda, and F. Egami. 1964. Studies of a pyocin. III. Biological properties of the pyocin. J. Biochem. (Tokyo) 55:59-64.

17. Nomura, M. 1967. Colicins and related bacteriocins. Annu. Rev. Microbiol. 21:257-284.

18. Roslycky, E. B. 1967. Bacteriocin production in the Rhizobia bacteria. Can. J. Microbiol. 13:431-432.
19. Simon, L. D., and T. F. Anderson. 1967. The infection of Escherichia coli by $\mathrm{T} 2$ and $\mathrm{T} 4$ bacteriophages as seen in the electron microscope. I. Attachment and penetration. Virology 32:279-297.

20. Simon, L. D., and T. F. Anderson. 1967. The infection of Escherichia coli by T2 and T4 bacteriophages as seen in the electron microscope. II. Structure and function of the baseplate. Virology 32:298-305.

21. Taubeneck, U. Über inkomplette Bakteriophagen aus defekt lysogenen Proteus mirabilis-Stämmen. Biol. Zentralbl. (Suppl.) 86:45-54. 